\title{
POSITIVE DISKS WITH PRESCRIBED MEAN CURVATURE ON THE BOUNDARY*
}

\author{
HONG JIAXING ${ }^{\dagger}$
}

1. Introduction. By a positive disk we mean a positive curvature metric $g=$ $g_{i j} d u^{i} d u^{j}$, defined on the closed unit disk $\bar{D}=\left\{\left(u^{1}\right)^{2}+\left(u^{2}\right)^{2} \leq 1\right\}$. In the sequel we always denote it by $(\bar{D}, g)$. Recently, there have been some papers devoted to the boundary value problems for isometric embedding of positive disks into $R^{3}$ with prescribed vertical component of position vector, for example, [PO] [DE] [HO2] and [HO3]. The present paper is devoted to the Neumann problem for isometric embedding of positive disks which is posed by Yau in [Y4]. Given a smooth positive disk $(\bar{D}, g)$ and a positive function $h \in C^{\infty}(\partial D)$, can we find a smooth surface

$$
\vec{r}: \bar{D} \mapsto R^{3} \text { such that } d \vec{r}^{2}=g
$$

with the prescribed mean curvature $h$ on $\vec{r}(\partial D)$

Before describing our results, we first introduce an invariant related to umbilical points of surfaces in $R^{3}$. Suppose that the given metric is of the form

$$
g=E d x^{2}+2 F d x d y+G d y^{2},(x, y) \in \bar{D} .
$$

Let $\vec{r}$ be a smooth isometric immersion of $(\bar{D}, g)$ with the second fundamental form

$$
I I=L d x^{2}+2 M d x d y+N d y^{2} \text { for }(x, y) \in \bar{D}
$$

DEFINITION. If $\vec{r}$ is of no umbilical points on $\partial D$, with

$$
\sigma=2(E M-F L)+\sqrt{-1}(G L-E N)
$$

the winding number of $\sigma$ on $\partial D$ is called the index of the umbilical points of the surface $\vec{r}$ and denoted by Index $(\vec{r})$ or Index $(\sigma)$.

Obviously, this definition makes sense since $p$ is an umbilical point if and only if $\sigma(p)=0$. We shall show that the definition of the index of the umbilical points is coordinate-free and hence, an invariant of describing umbilical points of surfaces.

The problem about the realization of a positive disk into $R^{3}$ seems to have some obstructions. For details, refer to Gromov's counter example [GR] which is an analytic positive disk not admitting any $C^{2}$ isometric immersion. On the other hand, to the author's knowledge the unique known sufficient condition for a smooth positive disk to be smoothly embedded into $R^{3}$ is the geodesic curvature of the boundary positive. Therefore the following hypothesis for the solvability is natural. Assume that

$$
(\bar{D}, g) \text { admits a smooth isometric immersion } \vec{r}_{0} \text { in } R^{3}
$$

The main result of the present paper is as follows.

THEOREM A. If $(\bar{D}, g)$ is a smooth positive disk satisfying (1.4), then for any nonnegative integer $n$ and arbitrary $(n+1)$ distinct points $p_{0} \in \partial D, p_{1}, \ldots, p_{n} \in D$,

\footnotetext{
* Received May 8, 2000; accepted for publication September 4, 2000.

$\dagger$ Institute of Mathematics, Fudan University, Shanghai, 200433, China (jxhong@fudan.ac.cn). Supported by the project of MSTC, the NSF of China, the STDF of Shanghai.
} 
the problem (1.1) admits two and only two solutions $\vec{r}$ in $C^{\infty}\left(\bar{D}, R^{3}\right)$ with prescribed mean curvature $h$ on $\partial D$ and moreover,

one principal direction at $p_{0}$ is tangent to $\partial D$, and at $p_{k}$ parallel to that of $\vec{r}_{0}$, besides, Index $(\vec{r})=n$ and $H\left(p_{k}\right)=H_{0}\left(p_{k}\right), k=1, \ldots, n$

where $H$ and $H_{0}$ are respectively the mean curvatures of $\vec{r}$ and $\vec{r}_{0}$ provided that

$$
\frac{h}{\sqrt{K}}-1>4 \max _{\partial D}\left[\frac{H_{0}}{\sqrt{K}}-1\right] \text { on } \partial D
$$

Besides, this solution is a convex surface if the geodesic curvature of $\partial D$ is nonnegative and

$$
\left(\theta+\sqrt{\theta^{2}-K}\right) d \leq \pi \text { where } \theta=\operatorname{Max}\left\{|h|_{\infty}, \sqrt{|K|_{\infty}+\left|K^{-1}\right|_{\infty}|\Delta K|_{\infty}}\right\}
$$

and $d$ is the diameter of the given positive disk, $\Delta$ is the Laplacian with respect to the given metric.

Throughout the present paper the uniqueness means unique up to a rigid motion in $R^{3}$. It is worth pointing out two extreme cases. The first one involves the existence. Suppose that the given positive disk $(\bar{D}, g)$ is of positive constant curvature. Then it is easy to see that this positive disk admits a smooth isometric embedding $\vec{r}_{0}$ in $R^{3}$ which is a simply connected region of the sphere. Under the present circumstance $\vec{r}_{0}$ is totally umbilical and hence, the right hand side of (1.6) vanishes. Therefore we have

THEOREM B. If $(\bar{D}, g)$ is of constant curvature and $\sqrt{K}<h \in C^{\infty}(\partial D)$, then (1.1) with (1.5) is always solvable for each nonnegative integer $n$ and arbitrary $(n+1)$ distinct points $p_{0} \in \partial D, p_{1},, . ., p_{n} \in D$.

The second extreme case involves the nonexistence. If the given positive disk is radius symmetric, i.e., $g=d r^{2}+G^{2}(r) d \theta^{2} 0 \leq r \leq 1$ where $G \in C^{\infty}([0,1])$ and $G(0)=0, G^{\prime}(0)=1, G>0$ as $r>0$. Then if $G_{r}>-1,(\bar{D}, g)$ has such a smooth isometric embedding in $R^{3}$,

$$
\vec{r}_{0}: x=G(r) \cos \theta, y=G(r) \sin \theta, z=-\int_{r}^{1} \sqrt{1-G_{r}^{2}} d r
$$

With its mean curvature $H_{0}=H_{0}(r)$ we have

THEOREM C. If $H_{0}(1)>\sqrt{K(1)}$, then for arbitrary $h \in C^{\infty}(\partial D)$ satisfying $\sqrt{K(1)} \leq h<H_{0}(1)$ the problem (1.1) has no any $C^{2}$ solution.

It should be also pointed out that for this radius symmetric positive disk with the aid of Theorem A (1.1) with (1.5) always admits two and only two solutions in $C^{\infty}\left(\bar{D}, R^{3}\right)$ provided that $C^{\infty}(\partial D) \ni h>4 H_{0}-3 \sqrt{K}$ as $r=1$.

The sketch of the present paper is as follows. In Section 2 the invariance of the index of umbilical points is discussed. In Section 3 the openness part of the method of continuity is given and in Section 4 and Section 5 the proofs for Theorem A and Theorem $\mathrm{C}$ are completed.

2. The index of umbilical points. Let the smooth metric be given in the form of (1.2) and let $\vec{r}$ be its smooth isometric immersion with the second fundamental form (1.3).

Lemma 2.1. If $\sigma \neq 0$ on $\partial D$, then the index of umbilical points of the surface, Index $(\sigma)$ is coordinate-free. 
Proof. We first use the isothermal coordinates of the induced metric of the surface, namely, $g=E\left(d x^{2}+d y^{2}\right),(x, y) \in \vec{D}$ for some smooth positive function $E$. Hence, in the present case,

$$
\sigma=2 M E+i(L-N) E \neq 0 \text { on } \partial D
$$

Suppose that there is a $C^{1}$ diffeomorphism from $(x, y) \ni \bar{D}$ onto $(\xi, \eta) \in \bar{D}$ and that in the new coordinates the first and second fundamental forms $g=\tilde{E} d \xi^{2}+2 \tilde{F} d \xi d \eta+\tilde{G} d \eta^{2}$, $I I=\tilde{L} d \xi^{2}+2 \tilde{M} d \xi d \eta+\tilde{N} d \eta^{2}$ respectively. where

$$
\begin{aligned}
& \tilde{E}=E\left(x_{\xi}^{2}+y_{\xi}^{2}\right), \tilde{G}=E\left(x_{\eta}^{2}+y_{\eta}^{2}\right) \text { and } \tilde{F}=E\left(x_{\xi} x_{\eta}+y_{\xi} y_{\eta}\right) \\
& \tilde{L}=L x_{\xi}^{2}+2 M x_{\xi} y_{\xi}+N y_{\xi}^{2}, \tilde{N}=L x_{\eta}^{2}+2 M x_{\eta} y_{\eta}+N y_{\eta}^{2} \text { and } \\
& \tilde{M}=L x_{\xi} x_{\eta}+M\left(x_{\xi} y_{\eta}+x_{\eta} y_{\xi}\right)+N y_{\xi} y_{\eta}
\end{aligned}
$$

Consequently, by the definition,

$$
\begin{aligned}
\tilde{\sigma} & =E J(2 A+i B) \text { where } J=\operatorname{det}\left(\frac{\partial(x, y)}{\partial(\xi, \eta)}\right), \\
A & =(N-L) x_{\xi} y_{\xi}+M\left(x_{\xi}^{2}-y_{\xi}^{2}\right), \\
B & =(L-N)\left(x_{\eta} y_{\xi}+x_{\xi} y_{\eta}\right)+2 M\left(y_{\xi} y_{\eta}-x_{\xi} x_{\eta}\right)
\end{aligned}
$$

Expressing them in terms of matrix we have

$$
T\left(\begin{array}{c}
M \\
L-N
\end{array}\right)=\left(\begin{array}{cc}
\left(x_{\xi}^{2}-y_{\xi}^{2}\right) & -x_{\xi} y_{\xi} \\
2\left(y_{\xi} y_{\eta}-x_{\xi} x_{\eta}\right) & \left(x_{\eta} y_{\xi}+x_{\xi} y_{\eta}\right)
\end{array}\right)\left(\begin{array}{c}
M \\
L-N
\end{array}\right)=\left(\begin{array}{c}
A \\
B
\end{array}\right)
$$

Notice that $\operatorname{det}(T)=\left(x_{\xi}^{2}+y_{\xi}^{2}\right)\left(x_{\xi} y_{\eta}-x_{\eta} y_{\xi}\right)$ equals zero nowhere on $\bar{D}$. This implies that $\tilde{\sigma}=E J(2 A+i B)$ vanishes nowhere on $\partial D$ from (2.1) and hence, Index $(\tilde{\sigma})$ is well defined.

Since the winding number is invariant under the homotopy preserving $\sigma \neq 0$ on $\partial D$, we can make such a homotopy $T_{\lambda}, \lambda \in[0,1]$

$$
\begin{aligned}
& x_{\xi}^{\lambda}=x_{\xi}, y_{\xi}^{\lambda}=\frac{\left(x_{\xi} y_{\xi}+x_{\eta} y_{\eta}\right)}{x_{\xi}^{2}+x_{\eta}^{2}} \lambda x_{\xi}-\frac{J}{x_{\xi}^{2}+x_{\eta}^{2}} x_{\eta} \\
& x_{\eta}^{\lambda}=x_{\eta}, y_{\eta}^{\lambda}=\frac{\left(x_{\xi} y_{\xi}+x_{\eta} y_{\eta}\right)}{x_{\xi}^{2}+x_{\eta}^{2}} \lambda x_{\eta}+\frac{J}{x_{\xi}^{2}+x_{\eta}^{2}} x_{\xi}
\end{aligned}
$$

Replace $y_{\xi}$ and $y_{\eta}$ by $y_{\xi}^{\lambda}$ and $y_{\eta}^{\lambda}$ in (2.6) and then denote $T$ by $T_{\lambda}$. Set $\left(A_{\lambda}, B_{\lambda}\right)^{t}=$ $T_{\lambda}(M, L-N)^{t}$ and $\sigma_{\lambda}=E J\left(2 A_{\lambda}+\sqrt{-1} B_{\lambda}\right)$. Evidently, $\sigma_{1}=\tilde{\sigma}$ and $T_{\lambda}$ is continuous in $\vec{D} \times[0,1]$. Moreover,

$$
\operatorname{det}\left(T_{\lambda}\right)=\left[x_{\xi}^{2}+\left(y_{\xi}^{\lambda}\right)^{2}\right]\left(x_{\xi} y_{\eta}-x_{\eta} y_{\xi}\right) \neq 0 \text { on } \bar{D}
$$

Therefore $\operatorname{Index}\left(\sigma_{\lambda}\right)$ is also well defined for each $\lambda \in[0,1]$ and furthermore, $\operatorname{Index}\left(\sigma_{1}\right)=\operatorname{Index}\left(\sigma_{0}\right)$. With $\left|D_{\xi}\right|^{2}=x_{\xi}^{2}+y_{\xi}^{2}$ we have

$$
\left(\begin{array}{c}
A_{0} \\
B_{0}
\end{array}\right)=T_{0}\left(\begin{array}{c}
M \\
L-N
\end{array}\right)=\left(\begin{array}{cc}
\left(x_{\xi}^{2}-\frac{J^{2}}{\left|D_{\xi}\right|^{4}} x_{\eta}^{2}\right) & \frac{J}{\left|D_{\xi}\right|^{2}} x_{\xi} x_{\eta} \\
-2\left(1+\frac{J^{2}}{\left|D_{\xi}\right|^{4}}\right) x_{\xi} x_{\eta} & \frac{J}{\left|D_{\xi}\right|^{2}}\left(x_{\xi}^{2}-x_{\eta}^{2}\right)
\end{array}\right)\left(\begin{array}{c}
M \\
L-N
\end{array}\right)
$$


Construct another homotopy in such a way that first by the rotation we find $\left(c_{\lambda}, d_{\lambda}\right)$ $\in C^{0}(\bar{D} \times[0,1])$ subject to $\left(c_{0} \cdot d_{0}\right)=\left(x_{\xi}, x_{\eta}\right),\left(c_{1} \cdot d_{1}\right)=(1,0) \sqrt{\left(x_{\xi}^{2}+x_{\eta}^{2}\right)}$ and $c_{\lambda}^{2}+d_{\lambda}^{2}$ $=\left(x_{\xi}^{2}+x_{\eta}^{2}\right)$. Set

$$
\tilde{T}_{\lambda}=\left(\begin{array}{cc}
\left(c_{\lambda}^{2}-\frac{J^{2}}{\left|D_{\xi}\right|^{4}} d_{\lambda}^{2}\right) & \frac{J}{\left|D_{\xi}\right|^{2}} c_{\lambda} d_{\lambda} \\
-2\left(1+\frac{J^{2}}{\left|D_{\xi}\right|^{4}}\right) c_{\lambda} d_{\lambda} & \frac{J}{\left|D_{\xi}\right|^{2}}\left(c_{\lambda}^{2}-d_{\lambda}^{2}\right)
\end{array}\right)
$$

and define $\left(\tilde{A}_{\lambda}, \tilde{B}_{\lambda}\right)^{t}=\tilde{T}_{\lambda}(M, L-N)^{t}$. Similarly $\operatorname{det}\left(\tilde{T}_{\lambda}\right) \neq 0$ on $\bar{D}$. By the homotopy invariance we have

$$
\begin{aligned}
\operatorname{Index}(\tilde{\sigma}) & =\operatorname{Index}\left(\sigma_{0}\right) \\
& =\operatorname{Index}\left\{E J\left(x_{\xi}^{2}+x_{\eta}^{2}\right)\left[2 M+i \frac{J}{\left|D_{\xi}\right|^{2}}(L-N)\right]\right\} \\
& =\operatorname{Index}\{[2 M+i(L-N)]\}
\end{aligned}
$$

Comparison (2.9) with (2.1) soon completes the proof for the present lemma.

Now let us look at some surfaces.

$\operatorname{Index}(\sigma)=0$. Consider the surface

$$
z=x^{2}+2 y^{2}
$$

Then

$$
\begin{gathered}
E=1+4 x^{2}, F=8 x y, G=1+16 y^{2} \\
L=\frac{2}{D_{0}}, M=0, N=\frac{4}{D_{0}} \text { where } D_{0}=\sqrt{1+4 x^{2}+16 y^{2}}
\end{gathered}
$$

It is easy to see $G L-E N=-2$ at $x=y=0$ and hence, $G L-N E<0$ near $p=(0,0)$. Therefore for the given surface $B_{r}:\left(x, y, x^{2}+2 y^{2}\right), x^{2}+y^{2} \leq r^{2}$ where $\mathrm{r}$ is sufficiently small, the index of umbilical points on $\partial B_{r}, \operatorname{Index}(\sigma)=0$.

Index $(\sigma)=2$. Consider the surface

$$
z=\frac{1}{2}\left(x^{2}+y^{2}\right)+a x^{2} y^{2}\left(a>\frac{1}{4}\right)
$$

Then

$$
\begin{gathered}
E=1+x^{2}\left(1+2 a y^{2}\right)^{2}, F=x y\left(1+2 a x^{2}\right)\left(1+2 a y^{2}\right), G=1+y^{2}\left(1+2 a x^{2}\right)^{2} \\
L=\frac{1+2 a y^{2}}{D_{0}}, M=\frac{4 a x y}{D_{0}}, N=\frac{1+2 a x^{2}}{D_{0}}
\end{gathered}
$$

where $D_{0}=\sqrt{1+x^{2}\left(1+2 a y^{2}\right)^{2}+y^{2}\left(1+2 a x^{2}\right)^{2}},(x, y)=(0,0)$ is an isolated umbilical point if $a>1 / 4$ and

$$
\begin{gathered}
\text { Index }(\sigma)=\text { Index }\left[(4 a-1) x y+i(2 a+1)\left(y^{2}-x^{2}\right)\right] \\
=\operatorname{Index}\left(x^{2}-y^{2}+i 2 x y\right)=2 \text { as } a>\frac{1}{4}
\end{gathered}
$$


LEMMA 2.2. If the revolution surface $\vec{r}: z=f\left(\frac{1}{2} r^{2}\right), r=\sqrt{x^{2}+y^{2}} \leq 1$ has no umbilical point at $r=1$, then Index $(\vec{r})=-2$

Proof. A computation yields

$$
\begin{aligned}
& E=1+\left(f^{\prime}\right)^{2} x^{2}, F=\left(f^{\prime}\right)^{2} x y, G=1+\left(f^{\prime}\right)^{2} y^{2} \\
& L=\frac{f^{\prime}+f^{\prime \prime} x^{2}}{D_{0}}, M=\frac{f^{\prime \prime} x y}{D_{0}}, N=\frac{f^{\prime}+f^{\prime \prime} y^{2}}{D_{0}}
\end{aligned}
$$

where $D_{0}=\sqrt{1+\left(f^{\prime}\right)^{2}\left(x^{2}+y^{2}\right)}$. So

$$
\sigma=\frac{1}{D_{0}}\left\{\left[f^{\prime \prime}-\left(f^{\prime}\right)^{3}\right]\left(2 x y+i\left(x^{2}-y^{2}\right)\right\} \text { and } \operatorname{Index}(\sigma)=-2\right.
$$

since its mean curvature $H \neq \sqrt{K}$ implies $f^{\prime \prime}-\left(f^{\prime}\right)^{3} \neq 0$ as $r=1$.

3. Perturbation of the boundary value problem. Let $g=g_{i j} d u^{i} d u^{j}$ be a positive disk. Sometimes denote $u^{1}$ and $u^{2}$ by "1" and "2" or by $u$ and $v$. Let $\vec{r}$ be a smooth solution to the problem (1.1) and let its second fundamental form $I I=L d u^{2}+2 M d u d v+N d v^{2}$. Then $L, M$ and $N$ satisfy the Gauss-Codazzi equations

$$
\begin{aligned}
& L_{2}-M_{1}-\Gamma_{12}^{1} L-\left(\Gamma_{12}^{2}-\Gamma_{11}^{1}\right) M+\Gamma_{11}^{2} N=0 \\
& M_{2}-N_{1}-\Gamma_{22}^{1} L-\left(\Gamma_{22}^{2}-\Gamma_{12}^{1}\right) M+\Gamma_{12}^{2} N=0 \\
& L N-M^{2}=K \operatorname{det}\left(g_{i j}\right)=K|g|
\end{aligned}
$$

Next we try to put the Gauss-Codazzi equation in a complex form. Introduce the complex derivatives

$$
\partial_{z}=\frac{1}{2}\left(\partial_{u}-\sqrt{-1} \partial_{v}\right), \partial_{\bar{z}}=\frac{1}{2}\left(\partial_{u}+\sqrt{-1} \partial_{v}\right)
$$

Solving $N$ from (3.3) and substituting it into (3.1) (3.2) we can get a 2 X2 elliptic system. Introduce the complex Riemann invariants, as one has done in the case of the hyperbolic system (for negative Gaussian curvature)

$$
W=\frac{-M+i \sqrt{K|g|}}{L}
$$

Obviously,

$$
L=\frac{2 i \sqrt{K|g|}}{W-\bar{W}}, M=-i \frac{W+\bar{W}}{W-\bar{W}} \sqrt{K|g|}, N=\frac{2 i \sqrt{K|g|}}{W-\bar{W}}|W|^{2}
$$

We claim that $W$ satisfies the following first order complex equation:

$$
W_{2}+\bar{W} W_{1}=\tilde{F}(W, \bar{W}, g) \text { in } D
$$

where

$$
\begin{aligned}
\tilde{F}= & -\left[\Gamma_{22}^{1}+\left(\Gamma_{22}^{2}-\Gamma_{12}^{1}\right) \frac{W+\bar{W}}{2}-\Gamma_{12}^{2}|W|^{2}\right]-W\left[\Gamma_{12}^{1}+\left(\Gamma_{12}^{2}-\Gamma_{11}^{1}\right) \frac{W+\bar{W}}{2}-\Gamma_{11}^{2}|W|^{2}\right] \\
& +\frac{W-\bar{W}}{2}\left[\partial_{2}(\ln \sqrt{K|g|})+W \partial_{1}(\ln \sqrt{K|g|})\right]
\end{aligned}
$$

Indeed,

$$
W_{2}=\frac{-M_{2}+(\sqrt{K|g|})_{2} i}{L}-W \frac{L_{2}}{L} \text { and } \frac{N_{1}}{L}=W_{1} \bar{W}+W \frac{-M_{1}-(\sqrt{K|g|})_{1} i}{L}
$$


Combining (3.1)(3.3) with the last two expressions we can obtain (3.5) at once. By the definition of the complex derivatives inserting $\partial_{u}=\partial_{z}+\partial_{\bar{z}}$ and $\partial_{v}=i\left(\partial_{z}-\partial_{\bar{z}}\right)$ into (3.5). Then it is reduced to the following form

$$
W_{\bar{z}}=\mu(W) W_{z}+F(W, \bar{W}, g)
$$

where

$$
\mu=\frac{i+\bar{W}}{i-\bar{W}} \text { and } F=-\frac{\tilde{F}}{i-\bar{W}}
$$

It is easy to see $|\mu|<1$ as long as $K|g|>0$ and $|\mu|$ is strictly less than one if $M, L$ and $1 / L$ are finite. Since

Let us deal with the boundary condition of the prescribed mean curvature $h$.

$$
\begin{aligned}
\text { mean curvature } & =\frac{G L-2 M F+N E}{2|g|} \\
& =L \frac{G-2 \frac{M}{L} F+\frac{N}{L} E}{2|g|} \\
& =\frac{i \sqrt{K}}{(W-\bar{W}) \sqrt{|g|}}\left[G+F(W+\bar{W})+|W|^{2} E\right]
\end{aligned}
$$

namely,

$$
G+F(W+\bar{W})+|W|^{2} E=-i \sqrt{|g|} \frac{H}{\sqrt{K}}(W-\bar{W})
$$

Under the isothermal coordinates the boundary condition is of the form

$$
1+|W|^{2}+i \frac{h}{\sqrt{K}}(W-\bar{W})=0 \text { on } \partial D
$$

Furthermore in the present case $F$ in the right hand side of (3.6) is subject to

$$
\left.F(W, \bar{W}, g)\right|_{W=i}=-\frac{i}{4} \partial_{z} \ln K
$$

Now we proceed to deal with the linearized problem of (3.6) (3.9). Suppose that the given surface $\vec{r}$ is a smooth solution to the problem (1.1). By the definition of $W$ in (3.4) and $\mu$ in (3.7) we have $|\mu| \leq \mu_{0}<1$ on $\bar{D}$ for some constant $\mu_{0}$. And the linearized problem of (3.6) (3.9) is as follows

$$
V_{\bar{z}}=\mu(W) V_{z}+A V+B \bar{V}+F_{0} \text { in } D
$$

with

$$
\operatorname{Re}\{\bar{\sigma} V\}=h_{0}, \text { as }|z|=1 \text { where } \sigma=i \frac{h}{\sqrt{K}}-W
$$

and $A, B$ are smooth functions depending only on the first and second fundamental forms of the surface $\vec{r}$. In view of (3.9), a direct computation gives

$$
|\sigma|^{2}=\frac{h^{2}}{K}-1>0 \text { on } \partial D \text { if } h>\sqrt{K} \text { on } \partial D
$$


Therefore the winding number of $\sigma$ along $\partial D, \operatorname{Index}(\sigma)$ is well defined. On the other hand, by the definition of $W$, we have

$$
\sigma=\frac{M}{L}+i\left\{\frac{L+N}{2 \sqrt{K} E}-\frac{\sqrt{K} E}{L}\right\}=\frac{M}{L}+i\left\{\frac{L^{2}-L N+2 M^{2}}{2 L \sqrt{K} E}\right\}
$$

Since $L, \sqrt{K}$ and $E$ are positive, it turns out

$$
\operatorname{Index}(\sigma)=\operatorname{Index}\{2 M+i(L-N)\}
$$

This is nothing else but the index of umbilical points of the surface $\vec{r}$ introduced in Section 1. If Index $(\sigma)=n$, it is easy to see $\sigma z^{-n}=|\sigma| \exp (\sqrt{-1}(\operatorname{Arg} \sigma-n \theta))$ on $\partial D$ where $(\operatorname{Arg} \sigma-n \theta)$ is a smooth function on $\partial D$. Since $|\sigma| \neq 0$, then without loss generality we may assume that $\sigma=z^{n}$, otherwise smoothly extend the domain of $|\sigma|$ and $(\operatorname{Arg} \sigma-n \theta)$ to $\bar{D}$ by preserving $|\sigma| \neq 0$, A change of unknown function $V$ to $V$ $|\sigma| \exp (-\sqrt{-1}(\operatorname{Arg} \sigma-n \theta))$ gives this situation. Now we are faced with the following boundary value problem (3.11) with

$$
\operatorname{Re}\left(z^{-n} V\right)=h_{0}
$$

By the Vekua's theory the solution of (3.11) with (3.14) is of the form

$$
V=T(f)+\Gamma\left(h_{0}\right)+\phi(z)
$$

Here

$$
\Gamma\left(h_{0}\right)=\frac{z^{n}}{2 \pi i} \int_{\partial D} h_{0} \frac{t+z}{t-z} \frac{d t}{t}, \phi(z)=i c_{n} z^{n}+\sum_{k=0}^{n-1}\left(c_{k} z^{2 n-k}-\bar{c}_{k} z^{k}\right)
$$

with complex constants $c_{k}, k=0,1, \ldots, n$ and $c_{n}$ real, and

$$
\begin{aligned}
T f & =-\frac{1}{\pi} \int_{D}\left[\frac{f(\zeta)}{\zeta-z}+\frac{z^{2 n+1} \bar{f}(\zeta)}{1-\bar{\zeta} z}\right] d \zeta \wedge d \bar{\zeta} \\
& =T_{0} f-\frac{1}{\pi} \int_{D} \frac{z^{2 n+1} \bar{f}(\zeta)}{1-\bar{\zeta} z} d \zeta \wedge d \bar{\zeta}
\end{aligned}
$$

is a bounded operator from $L^{p}(D)$ into $W^{1, p}(D)$ for all $p \in(1, \infty)$ and moreover, $\partial_{\bar{z}} T f=f$. Therefore, $f$ satisfies the following integral equation

$$
\begin{aligned}
& f-\mu\left[\partial_{z} T f\right]-A T f-B \bar{T} f \\
= & F_{0}+\mu\left[\partial_{z} \Gamma\left(h_{0}\right)+\partial_{z} \phi(z)\right]+A\left[\Gamma\left(h_{0}\right)+\phi\right]+B\left[\bar{\Gamma}\left(h_{0}\right)+\bar{\phi}\right]
\end{aligned}
$$

An important observation for this integral equation is, as an operator in $L^{2}(D)$,

$$
\Lambda_{2}=\sup _{f \in L^{2}(D)} \frac{\left\|\partial_{z} T f\right\|}{\|f\|} \leq 1
$$

(for details, see [V] ) and with $\Lambda_{p}$ the norm of operators in $L^{p}(D)$ by the convexity of $\log \Lambda_{p}$ in $p$ and the fact that $|\mu|_{\infty}$ is strictly less than one we know

$$
\Lambda_{p}|\mu|_{\infty}<1 \text { for some } p>2 \text { close to } 2
$$

Hence, the principal part of (3.18) is contractive and (3.18) can be reduced to a Fredholm operator in $L^{p}(D)$. In the present section, unless otherwise statement, $p$ always satisfies (3.20). 
Lemma 3.1. Let $A, B$ and $\mu$ be bounded measurable functions and $|\mu|_{\infty}<1$. Then if Index $(\sigma)=n \in Z^{+}$then the homogeneous problem of (3.11) (3.14) has no nontrivial solutions in $W^{1, p}(D)$ satisfying $V\left(p_{0}\right)=0$ and in addition, $V\left(p_{k}\right)=0$, $k=1, \ldots, n$ if $n \geq 1$ for arbitrary $p_{0} \in \partial D$ and distinct $p_{k} \in D$.

Proof. If $V$ is a solutions mentioned in this lemma. By the Vekua's theory [V] (also see Lemma 4.1 in the present paper) we can find two functions $\phi, \zeta \in W^{1, p}\left(R^{2}\right)$ respectively satisfying

$$
\begin{aligned}
& \phi_{\bar{z}}=\mu \phi_{z}+\left[A+B \frac{\bar{V}}{V}\right] \text { in } D \text { and } \operatorname{Im} \phi=0 \text { on } \partial D \\
& \zeta_{\bar{z}}=\mu \zeta_{z} \text { in } D
\end{aligned}
$$

where $\zeta$ is a homemorphism from $\bar{D}$ onto $\bar{D}$ with $\zeta(0)=0$ and $\zeta\left(p_{0}\right)=p_{0}$ (also see $[A])$ and moreover, both of $\phi$ and $\zeta$ are analytic outside disk. It is evidently that $V_{1}=$ $V \exp (-\phi)$ satisfies (3.22) in $D$ and a direct computation gives, as the function of $\zeta$, $V_{1}$ is analytic in $D$ and continuous on $\bar{D}$. Furthermore from (3.14) we have

$$
\left.\operatorname{Re}\left(z^{-n} e^{+\phi} V_{1}(\zeta)\right)=\operatorname{Re}\left(\zeta^{-n} \lambda_{1} V_{1}(\zeta)\right)\right)=0 \text { on } \partial D
$$

where $\lambda_{1}=e^{+\phi}(\zeta / z)^{n}$. It is easy to see, as the function of $\zeta, \operatorname{Index}\left(\lambda_{1}\right)=0$ on $\partial D$ since $\zeta$ is a homemorphism, preserving the origin and $p_{0}$. (3.23) tells us, for some function $q(\zeta)$ analytic in $D$ and continuous on $\bar{D}$ satisfying $\operatorname{Im} q=n(\arg (\zeta)-\arg (z))$ on $\partial D, \operatorname{Re}\left(\zeta^{-n} e^{q_{1}(\zeta)} V_{1}\right)=0$ on $\partial D$. Hence,

$$
e^{q_{1}(\zeta)} V_{1}=e^{q_{1}(\zeta)} V e^{-\phi}=i c_{n} \zeta^{n}+\sum_{k=0}^{n-1}\left(c_{k} \zeta^{2 n-k}-\bar{c}_{k} \zeta^{k}\right) \text { on } \bar{D}
$$

Without loss of generality we may assume $p_{1}=0$ and $p_{0}=(1,0)$. Hence $V\left(p_{1}\right)=0$ implies $c_{0}=0$ and

$$
\begin{aligned}
& i c_{n}+\sum_{k=1}^{n-1}\left(c_{k}-\bar{c}_{k}\right)=0 \\
& i c_{n} p_{m}^{n}+\sum_{k=1}^{n-1}\left(c_{k} p_{m}^{2 n-k}-\bar{c}_{k} p_{m}^{k}\right)=0, m=2, \ldots, n \\
& i c_{n}\left(\bar{p}_{m}\right)^{-n}+\sum_{k=1}^{n-1}\left(c_{k}\left(\bar{p}_{m}\right)^{-(2 n-k)}-\bar{c}_{k}\left(\bar{p}_{m}\right)^{-k}\right)=0, m=2, \ldots, n
\end{aligned}
$$

View of (3.24) (3.25) (3.26) as a linear algebraic equations with the unknowns $c_{1}, \ldots$, $c_{n-1}, i c_{n},-\bar{c}_{n-1},-\bar{c}_{n-2}, \ldots,-\bar{c}_{1}$. It is easy to see that the matrix of the coefficients is nothing else but the Vandermonde determinate composed of $1, p_{2}, \ldots, p_{n},\left(\bar{p}_{2}\right)^{-1}$, $\ldots,\left(\bar{p}_{n}\right)^{-1}$. Since $p_{k} \neq p_{k^{\prime}}$ and $p_{k} \in D$ for all $k \geq 1, c_{1}=c_{3}=, . .,=c_{n}=0$ follows immediately. This proves $V \equiv 0$ and completes the proof of the present lemma.

From the above argument it is easy to fix the constants $c_{k}$ in (3.16) such that

$$
\operatorname{Im}\left\{z^{-n} V\left(p_{0}\right)\right\}=l_{0}, V\left(p_{1}\right)=l_{1}, \ldots, V\left(p_{n}\right)=l_{n}
$$

where $l_{0} \in R^{1}, l_{1}, \ldots, l_{n}$ are given constants and moreover, uniquely

$$
c_{i}=L_{i}\left(l_{k}, T(f)\left(p_{k}\right), B\left(h_{0}\right)\left(p_{k}\right) \mid k=0,1, \ldots, n\right), i=0,1, \ldots, n
$$

where $L_{i}$ are some linear combinations of $l_{k}, T(f)\left(p_{k}\right), B\left(h_{0}\right)\left(p_{k}\right), k=0,1, \ldots, n$. 
LEMMA 3.2. If $F_{0} \in L^{p}(D), h_{0}=\gamma_{0}(\tilde{h})$ where $\tilde{h} \in W^{1, p}(D)$ and $\gamma_{0}$ is the trace operator, and the constants $c_{k}$ are as mentioned in (3.27), then (3.11) (3.14) always admits a solution $V \in W^{1, p}(D)$ and moreover,

$$
\|V\|_{W^{1, p}(D)} \leq C\left\{\|\tilde{h}\|_{W^{1, p}(D)}+\left\|F_{0}\right\|_{L p_{(D)}}+\sum_{k=0}^{n}\left|l_{k}\right|\right\}
$$

for some constant $C$ depending only on $|A|_{\infty},|B|_{\infty}$ and $1 /\left(1-|\mu|_{\infty}\right)$.

Proof. Notice that $T$ and $\Gamma$ are respectively the bounded operators from $L^{p}(D)$ and $W^{1, p}(D)$ into $W^{1, p}(D)$. (3.20) guarantees (3.18) of Fredholm type. Now its solvability is equivalent to the uniqueness of solutions to the homogeneous equation. If $f \in L^{p}(D)$ is a solution of (3.18) for $F_{0}=0, h_{0}=0$ and $l_{k}=0, k=0,1, \ldots, n$, it follows that

$$
V=T f+i c_{n} z^{n}+\sum_{k=0}^{n-1}\left(c_{k} z^{2 n-k}-\bar{c}_{k} z^{k}\right)
$$

satisfies the homogeneous problem of $(3.11)(3.14)$. On the other hand, by the construction of $c_{k}$ it follows $V\left(p_{0}\right)=0, V\left(p_{k}\right)=0, k=1, . . n$. Thus Lemma 3.1 tells us $V$ identically zero. In view of the fact that $\partial V / \partial \bar{z}=f$, we know $f$ and all constants $c_{k}$ also identically zero. This proves the uniqueness of solution to (3.18) in $L^{p}(D)$. (3.28) is an immediate consequence of the compact argument. This ends the proof for the present lemma.

We shall prove the main theorem of the present paper by the method of continuity. Consider an one-parameter family of smooth positive disks.

$$
g_{\lambda}=E_{\lambda}\left(d u^{2}+d v^{2}\right),(u, v) \in \bar{D} \text { for some } E_{\lambda} \in C^{1}\left([0,1], C^{\infty}(\bar{D})\right)
$$

Assume that

$$
K_{\lambda} \geq \frac{1}{C} \text { on } \bar{D} \text { for all } \lambda \in[0,1]
$$

Let $h_{\lambda} \in C^{1}\left([0,1], C^{\infty}(\partial D)\right)$ satisfy

$$
h_{\lambda}>\sqrt{K_{\lambda}} \text { on } \partial D
$$

Denote by $W_{\lambda}$ the corresponding complex Riemann invariants of the solution to the problem (1.1) for $\left(\bar{D}, g_{\lambda}\right)$. Set

$$
\begin{aligned}
& S=\left\{\lambda \in[0,1] \mid \text { for } g_{\lambda} \text { the problem }(1.1) \text { has a smooth solution } \vec{r}_{\lambda}\right. \\
& \text { satisfying } W_{\lambda}\left(p_{k}\right)=l_{k}(\lambda) \text { for some } l_{k} \in C^{1}[0,1], k=0,1, \ldots, n \\
&\text { to be specified and } \left.\operatorname{Index}\left(W_{\lambda}-\frac{h_{\lambda}}{\sqrt{K_{\lambda}}} i\right)=n\right\}
\end{aligned}
$$

Of course $l_{0}$ could not be arbitrary and must be compatibility with the prescribed mean curvature $h_{\lambda}$.

THEOREM 3.3. The set $S$ is open.

Proof. Suppose that $\lambda_{0} \in S$, namely, there is a smooth solution $\vec{r}$ to the problem (1.1) satisfying (3.32). Denote the corresponding complex Riemann invariants by $W$ satisfying (3.6) (3.9) for $g_{\lambda_{0}}$ and $h_{\lambda_{0}}$ as well as (3.32). Now we try to find such kind solution $W+\epsilon V$ to the problem (1.1) for $g_{\lambda}$ and $h_{\lambda}$. Then $V$ satisfies the problem (3.11) (3.12) for $F_{0}=\epsilon Q(\epsilon, \lambda, W, \bar{W}, V, \bar{V})(V, \bar{V})+\epsilon^{-1} \delta \lambda \tilde{F}_{0}(\lambda, W)$ and $h_{0}=$ 
$\epsilon^{-1} \delta \lambda \tilde{h}(\lambda)-\frac{1}{2} \epsilon|V|^{2}$ where $\delta \lambda=\lambda-\lambda_{0}$ and $Q$ is a quadratic form of $V$ and $\bar{V}$ smoothly depending on $u, v$ and its arguments, $\tilde{F}_{0}, \tilde{h}_{0}$ are also some smooth functions of $u, v$ and its arguments. From (3.32) we can find all the constants $l_{0}, l_{1}, \ldots, l_{n}$ in (3.27) and finally reduce to the integral equation of $V$ in (3.18). It is easy to find the solution $(f, V)$ to $(3.15)$ and (3.18). Indeed, to put each $(\tilde{f}, \tilde{V}) \in L^{p}(D) \times W^{1, p}(D)$ in the right hand side of (3.15) and (3.18), the special choice of all constants and by means of Lemma 3.2 we can get a solution $(f, V) \in L^{p}(D) \times W^{1, p}(D)$ to (3.15) (3.18). Taking $\epsilon=\sqrt{\left|\lambda-\lambda_{0}\right|}$ and using Lemma 3.2 we can prove this map to be defined in the unit ball of $L^{p}(D) \times W^{1, p}(D)$ into itself and contractive if $\epsilon=\sqrt{\left|\lambda-\lambda_{0}\right|}$ is very small. Therefore the fixed point $(f, V)$ is just the required solution. The theorem on the regularity of solutions to the Riemann-Hilbert problem tells us $(f, V) \in C^{\infty}(\bar{D})$. From the obtained complex Riemann invariants $W+\epsilon V$, one can find the corresponding coefficients of the second fundamental form, $L, M$ and $N$ which certainly satisfy the Gauss-Codazzi equations. An application of the fundamental theorem on Differential Geometry gives the expected solutions to (1.1) with (3.32) if $\left|\lambda-\lambda_{0}\right|$ is very small.

LEMMA 3.4. For all $\lambda \in S$, the mean curvature of the corresponding solutions $\vec{r}$

$$
H \leq \max \left(|h|_{\infty}, \sqrt{|K|_{\infty}+\left|K^{-1}\right|_{\infty}|\Delta K|_{\infty}}\right)
$$

where $\Delta$ is the Laplacian operator with respect to the metric $g$.

Proof. Suppose that the mean curvature $H$ attains its maximum at some point $p *$. Then if $p * \in \partial D$, then (3.33) is trivial and if $p * \in D$, by [P. $p p 89$ Theorem 1] and also by [Y3] we know $H(p *)$ bounded above by the second part of the right hand side of (3.33). This ends the proof for the present lemma.

4. Several lemmas. This section intends to deal with the a priori estimates for the bounds of the solutions $\left|\vec{r}_{\lambda}\right|_{k+\alpha}$ where $\lambda \in S$. The difficulties come from the occurrence of umbilical points near $\partial D$. We must show that $H^{2}-K$ has a positive lower bound for all $\vec{r}_{\lambda}$ where $\lambda \in S$ near $\partial D$. Before doing so we introduce some lemmas.

LEMMA 4.1. If $\mu \in C^{\infty}(\bar{D})$ and $|\mu|_{\infty}<1$, then there is a $\zeta(z) \in C^{\infty}(D)$ which is homeomorphism from $\bar{D}$ onto $\bar{D}$ with $\zeta(0)=0$ and satisfies

$$
\zeta_{\bar{z}}=\mu \zeta_{z} \text { in } D
$$

Moreover, its inverse map $z=z(\zeta)$ is in $C^{\infty}(D)$ and $\|D \zeta\|_{p},\|D z\|_{p} \leq C$ for some $p>2$ and some constant $C$ depending only on $1 /\left(1-|\mu|_{\infty}\right)$.

Proof. This is a result due to [V]. For convenience of readers the proof is given here. First of all we assume $\mu \in C^{\infty}\left(R^{2}\right)$ with supp $\mu \subset \bar{D}$. Let us look for such solutions of the form

$$
\zeta=z+T_{0} f-\left(T_{0} f\right)(0)
$$

where $T_{0}$ is the Vekua's operator mentioned in (3.17) and $\zeta$ satisfies

$$
f=\mu \Pi f+\mu \text { in } L^{p}\left(R^{2}\right)
$$

where $\Pi=\partial_{z} T_{0}$. From the assumption $|\mu|_{\infty}<1$ and the properties of operator $\Pi$ it is easy to see that (4.3) admits a solution $f \in L^{p}\left(R^{2}\right)$ where $p \in(2,2+\epsilon)$ for some positive constant $\epsilon$ and $|\mu|_{\infty}\|\Pi\|_{p}<\left(1+|\mu|_{\infty}\right) / 2$. Hence $\|f\|_{p} \leq 2 /\left(1-|\mu|_{\infty}\right)$ and $\zeta \in W^{1, p}(D),\|D \zeta\|_{p}$ is controlled by $1 /\left(1-|\mu|_{\infty}\right)$. On the other hand, since $\zeta=z(1+0(1 /|z|))$ as $z \mapsto \infty$, it follows that for each point $c_{0} \in C^{1}, \zeta-c_{0} \neq 0$ in $R^{2} \backslash B_{r}(0)$ if $\mathrm{r}$ is sufficiently large and the winding number of $\zeta-c_{0}$ on $\partial B_{r}(0)$ equals 
one. By the argument principle on the generalized analytic function there is only one null point in $B_{r}(0)$. So $\zeta$ is homeomorphic from $R^{2}$ onto $R^{2}$. By the regularity theory on Beltrami equation we know $\zeta \in C^{\infty}\left(R^{2}\right)$ and $D \zeta \neq 0$ in $R^{2}$. Thus we have proved $\zeta$ diffeomorphic from $\bar{D}$ onto $\zeta(\bar{D})$. Composition a conformal mapping with $\zeta$ soon gives a diffeomorphism from $\bar{D}$ onto $\bar{D}$, satisfying all the statement of the present lemma for $\zeta$. Let us now estimate the norm of its inverse map $z=z(\zeta)$ in $W^{1, p}(D)$. A direct computation gives

$$
z_{\bar{\zeta}}=-\mu(z(\zeta)) \bar{z}_{\bar{\zeta}}
$$

Therefore $z=\zeta+T_{0} h+\phi(\zeta)$ where $h=-\mu \bar{z}_{\bar{\zeta}} \in C^{\infty}(\bar{D})$ and $\phi$ analytic in $R^{2}$. Combining (4.2) with (4.4) tells us $\phi(\zeta)$ bounded in the whole complex plane. This implies $\phi(\zeta)$ is the constant $-T_{0} h(0)$. Inserting $\mathrm{z}$ into (4.3) we have

$$
h=-\mu(z(\zeta)) \bar{\Pi} h-\mu(z(\zeta)) \text { in } L^{p}(D)
$$

Analogously we can soon get, as a function of $\zeta, z=z(\zeta) \in W^{1, p}(D)$ and $\|D z\|_{p} \leq$ $2 /\left(1-|\mu|_{\infty}\right)$.

Finally a limit procedure will soon complete the proof for the present lemma.

Now let us consider a special problem

$$
\begin{aligned}
& W_{\bar{z}}=\mu W_{z} \text { in } D \text { with }|W|=1 \text { on } \partial D \\
& \left.W\left(p_{0}\right)=i \text { (or }-i\right) \\
& \text { Index }(W)=n, \text { and } W\left(p_{k}\right)=\xi, k=1,2, \ldots, n
\end{aligned}
$$

where $|\xi|<1$ and $p_{0} \in \partial D, p_{k} \in D, k=1,2, \ldots n$ are distinct.

LEMMA 4.2. Suppose that $\mu$ is a smooth function of $W, \bar{W}, z, \bar{z}$ and $\sup |\mu| \leq$ $q<1$ where the supremum is taken over all $|z| \leq 1$ and $|W| \leq 1$ for some constant $\bar{q}$. Then (4.6) with (4.7)(4.8) always admits a unique solution in $C^{\infty}(\bar{D})$.

Proof. It suffices to prove Lemma 4.2 for the case $W\left(p_{0}\right)=i$. Replace $\mu$ by $t \mu$. Then at $t=0$ we have a unique analytic solution

$$
\frac{W-\xi}{1-\bar{\xi} W}=e^{i \alpha} \frac{z-p_{1}}{1-\bar{p}_{1} z} \frac{z-p_{2}}{1-\bar{p}_{2} z} \ldots . \frac{z-p_{n}}{1-\bar{p}_{n} z}
$$

Choose the constant $\alpha$ so that $W\left(p_{0}\right)=i$. It is easy to see that the following set

$$
\Lambda=\left\{t \in[0,1] \mid(4.6)-(4.8) \text { have a solution in } C^{\infty}(\bar{D})\right\}
$$

is not empty. By the same argument as that in proving Theorem 3.3 we can see $\Lambda$ open. By the maximum principle $|W|_{\infty} \leq 1$ for each solution $W$ to (4.6)-(4.8). Hence $|\mu|_{\infty} \leq q<1$. Suppose that $\zeta=\zeta_{t}(z)$ and $z=z_{t}(\zeta)$ are the homeomorphism mentioned in Lemma 4.1 for $t \mu$. It is evident that $\zeta_{t}(z)\left(z_{t}(\zeta)\right)$ are equicontinuous for all $t \in \Lambda$. Set $\zeta_{t}\left(p_{k}\right)=p_{k}(t)$. It is easy to see that there is a positive constant $\delta_{1}$ independent of $t \in \Lambda$ such that $\left|p_{k}(t)\right| \leq 1-\delta_{1}$. Since $W$ is analytic in $\zeta \in D$, it follows that $W$ has the explicit formula

$$
\frac{W-\xi}{1-\bar{\xi} W}=e^{i \alpha} \frac{\zeta-\zeta\left(p_{1}(t)\right)}{1-\bar{\zeta}\left(p_{1}(t)\right) \zeta} \frac{\zeta-\zeta\left(p_{2}(t)\right)}{1-\bar{\zeta}\left(p_{2}(t)\right) \zeta} \ldots \frac{\zeta-\zeta\left(p_{n}(t)\right)}{1-\bar{\zeta}\left(p_{n}(t)\right) \zeta}
$$

Therefore there is another positive constant $\delta$ also independent of $t \in \Lambda$ such that $|W| \geq \frac{1}{2}$ as $|\zeta| \geq 1-\delta$. With $\phi=|W|^{2}$ we have

$$
\phi_{\zeta \bar{\zeta}}=\frac{\left|\phi_{\zeta}\right|^{2}}{\phi} \text { in } D \backslash \bar{D}_{\delta} \text { with } \phi=1 \text { on } \partial D
$$


and

$$
W_{\zeta}=\frac{\phi_{\zeta}}{\bar{W}}
$$

Now with the aid of Lemma 3.2 the interior estimates for the equation (4.6) yields, with $D_{\delta / 2}=\{p \in D \mid \operatorname{dis}(p, \partial D)>\delta / 2\}$

$$
\left\|D^{2} W\right\|_{L} p_{\left(D_{\delta / 2}\right)} \leq C_{p} \text { and }|D W|_{C \alpha_{\left(\bar{D}_{\delta / 2}\right)}} \leq C_{\alpha}
$$

for each $p \in[2,+\infty)$ and $\alpha \in(0,1)$ and some constants $C_{p}, C_{\alpha}$ depending only on $1 /\left(1-|\mu|_{\infty}\right)$ and $|\mu|_{1}$

Now we are in the position to deal with the estimates in $D \backslash D_{\delta}$. An application of the De Giorge' iteration to (4.12) with (4.13) and (4.14) soon bounds the Holder norm of $\phi$ in $C^{\beta}\left(\bar{D} \backslash D_{\delta}\right)$ for some positive constant $\beta$ depending only on $1 /(1-q)$ and hence, bounds the $L^{p_{-}}$norms over $D \backslash D_{\delta}$ for $D \phi$ as a function of $\zeta$ for each $p \in(2,+\infty)$. Pulling back to the original $z$ we have controlled the $L^{p}$-norm of $D W$ over $D$ for some $p>2$ close to 2 like in (3.20) and hence, the norm of $W$ in $C^{\alpha}(\bar{D})$ where $\alpha=1-2 / p$. Finally by differentiation of (4.6) and using the explicit formula (3.15) (3.18) without difficulty we can bound all the following norms

$$
\left\|D^{k} W\right\|_{L} p_{(D)} \leq C_{k p} \text { and }\left|D^{k} W\right|_{C} \alpha_{(\bar{D})} \leq C_{k \alpha}
$$

for each $p \in(2,+\infty)$ and each $\alpha \in(0,1)$ and for some constants $C_{k p}$ and $C_{k \alpha}$ under control. This proves $\Lambda$ closed.

Now let us prove the uniqueness. Suppose that there are two solutions to (4.6)(4.8), $W_{1}, W_{2}$. Instead of $\mu$ by $t \mu$ by means of the method of continuity previously done we can find two solutions $W_{1}(t), W_{2}(t) \in C\left([0,1], C^{\infty}(\bar{D})\right)$ with $W_{i}(1)=W_{i}$ $i=1,2$. Since at $t=0$, the problem (4.6) with (4.7) (4.8) is of the uniqueness, i.e., $W_{1}(0)=W_{2}(0)$ and $\Delta W=W_{1}(t)-W_{2}(t)$ satisfies

$$
(\Delta W)_{\bar{z}}=t \mu\left(W_{1}\right)(\Delta W)_{z}+t A_{1}(\Delta W)+t A_{2}(\Delta \bar{W}) \text { in } D \text { with }
$$

$$
\operatorname{Re}\left(\bar{W}_{1} \Delta W\right)=\frac{1}{2}|\Delta W|^{2}, \text { and } \Delta W\left(p_{0}\right)=0, \Delta W\left(p_{k}\right)=0, k=1,2, \ldots, n
$$

In view of the fact that Index $\left(W_{1}\right)=n$, a similar argument in proving Lemma 3.1 using the contraction mapping principle soon yields $\Delta W \equiv 0$ as $t \in\left[0, t_{0}\right]$ for some positive constant $t_{0}$. Step by step we can arrive at $t=1$ and conclude that $W_{1}(1)-$ $W_{2}(1)=W_{1}-W_{2}$ identically zero. This completes the proof for the present lemma.

Now scaling the given metric $g$ we have, in the isothermal coordinates,

$$
g_{\lambda}=\lambda^{2} E^{2}(\lambda u, \lambda v)\left(d u^{2}+d v^{2}\right),(u, v) \in \bar{D}
$$

Smoothly extend the domain of the given prescribed mean curvature $h$ to $\bar{D}$ such that

$$
\frac{h}{\sqrt{K}}-1>4 \max _{|u|=\lambda}\left\{\frac{H_{0}}{\sqrt{K}}-1\right\} \text { as }|u|=\lambda \text { for all } \lambda \in[0,1]
$$

and

$$
h(0)>\sqrt{K(0)} \frac{1+\left|W_{0}(0)\right|^{2}}{2 \operatorname{Im} W_{0}(0)}
$$

where $H_{0}$ and $W_{0}$ are respectively the mean curvature and the complex Riemann invariants of $\vec{r}_{0}$. This is possible by the hypothesis (1.6). Now we can fix the constants 
$l_{k}$ in $(3.32)$

$$
W_{\lambda}\left(p_{k}\right)=l_{k}=W_{0}\left(\lambda p_{k}\right) k=1, \ldots, n
$$

and

$$
W_{\lambda}\left(p_{0}\right)=i\left[\frac{h\left(\lambda p_{0}\right)}{\sqrt{K\left(\lambda p_{0}\right)}} \pm \sqrt{\frac{h^{2}\left(\lambda p_{0}\right)}{K\left(\lambda p_{0}\right)}-1}\right] \text { where } p_{0}=(1,0)
$$

Lemma 4.3. For each $n \in Z^{+}=\{0,1, . .$,$\} there is a constant \lambda_{0}$ and $W_{i}(\lambda) \in$ $C^{1}\left([0,1], C^{\infty}(\bar{D})\right) i=1,2$ which are the complex Riemann invariants of the solutions to the problem (1.1) for $\left(\bar{D}, g_{\lambda}\right) \lambda \in\left(0, \lambda_{0}\right]$ with the prescribed mean curvature $h(\lambda u)$ on $\partial D$ and satisfying (3.32) (4.19) and moreover, at $\lambda=0,\left(W_{i}-\sigma \sqrt{-1}\right) / \sqrt{\sigma^{2}-1}$ with $\sigma=h(0) / \sqrt{K(0)}$ are the solutions mentioned in Lemma 4.2.

Proof. Let us focus our attention on (3.6). In the present case, the corresponding $F$ subject to

$$
\begin{aligned}
& |i-\bar{W}|\left|F\left(W, \bar{W}, g_{\lambda}\right)\right| \leq \lambda C\left(|W|^{3}+1\right) \\
& |i-\bar{W}|^{2}\left|F_{W}\left(W, \bar{W}, g_{\lambda}\right)\right|,\left|F_{\bar{W}}\left(W, \bar{W}, g_{\lambda}\right)\right| \leq \lambda C\left(|W|^{3}+1\right)
\end{aligned}
$$

for some constant $C$ independent of $\lambda$ and $W, \bar{W}$. Similarly, by (3.9) the boundary condition is of the form

$$
1+|W|^{2}-2 \frac{h(\lambda u)}{\sqrt{K(\lambda u)}} \operatorname{Im} W=0 \text { on } \partial D
$$

When $\lambda=0,(4.21)$ is reduced to $|W-\sigma i|^{2}=\sigma^{2}-1$ on $\partial D$ with $\sigma=h(0) / \sqrt{K(0)}$. Consequently, if still denote $(W-\sigma i) / \sqrt{\sigma^{2}-1}$ by $W$, then $W$ is a solution to the following problem

$$
\begin{gathered}
\partial_{\bar{z}} W=\mu\left(\sqrt{\sigma^{2}-1} W+\sigma i\right) \partial_{z} W \text { in } D \text { with }|W|=1 \text { on } \partial D \\
W\left(p_{0}\right)= \pm i, \operatorname{Index}(W)=n
\end{gathered}
$$

and

$$
W\left(p_{k}\right)=\frac{W_{0}(0)-\sigma i}{\sqrt{\sigma^{2}-1}}=\xi, k=1, \ldots, n
$$

(4.18) guarantees $|\xi|<1$. It suffices to deal with the case $W\left(p_{0}\right)=i$. In view of (3.7), if $|W| \leq 1$, it turns out, $\operatorname{Im}\left[\sigma i+\sqrt{\sigma^{2}-1} \bar{W}\right] \geq \sigma-\sqrt{\sigma^{2}-1}$ and

$$
\mu\left(\sqrt{\sigma^{2}-1} W+\sigma i\right)=\frac{i+\left\{-\sigma i+\sqrt{\sigma^{2}-1} \bar{W}\right\}}{i+\left\{\sigma i-\sqrt{\sigma^{2}-1} \bar{W}\right\}}
$$

and hence,

$$
\left|\mu\left(\sqrt{\sigma^{2}-1} W+\sigma i\right)\right|_{\infty}^{2} \leq 1-\frac{4\left(\sigma-\sqrt{\sigma^{2}-1}\right)}{\left(\sigma+1+\sqrt{\sigma^{2}-1}\right)^{2}}
$$

Now the requirements in Lemma 4.2 are satisfied and the problem (4.22) (4.23) always admits a unique solution in $C^{\infty}(\bar{D})$. Pulling back to the original $W$ we have that when $\lambda=0$, for each $n \in Z^{+}$(3.6) with (3.9) admits a unique smooth solution satisfying (4.8) and $\operatorname{Im} W>0$ on $\bar{D}$.

When $\lambda>0$, in view of (4.20) and using the contraction mapping principle one can obtain that there is a positive constants $\lambda_{0}$ such that for all $\lambda \in\left[0, \lambda_{0}\right]$ the problem (3.6) with $(4.21)(3.32)(4.19)$ admits a smooth solution $W=W(\lambda)$ and moreover, 
$W(\lambda)$ is in $C^{1}\left(\left[0, \lambda_{0}\right], C^{\infty}(\bar{D})\right)$ and hence, $\operatorname{Im} W(\lambda) \geq C_{0}$ for some positive constant $C_{0}$ if $\lambda_{0}$ is small enough. This ends the proof of the present lemma.

5. Existence and uniqueness. This section intends to complete the proof of the main theorem in the present paper. Let us first derive an equation satisfied by $\frac{H}{\sqrt{K}}-1$. Under the isothermal coordinates, from (3.8) we have

$$
\frac{H}{\sqrt{K}}-1=\frac{1+|W|^{2}+i(W-\bar{W})}{-i(W-\bar{W})} . \text { Set } \Phi(W)=\frac{W-i}{\sqrt{-i(W-\bar{W})}}
$$

It is easy to see $|\Phi|^{2}=\frac{H}{\sqrt{K}}-1$ and a direct computation yields

$$
\Phi_{\bar{z}}=\frac{A \mu W_{z}-i(W-i) \bar{W}_{\bar{z}}+A F}{2[-i(W-\bar{W})]^{3 / 2}}
$$

and

$$
\Phi_{z}=\frac{A W_{z}-i(W-i) \bar{\mu} \bar{W}_{\bar{z}}-i(W-i) \bar{F}}{2[-i(W-\bar{W})]^{3 / 2}} \text { where } A=i(2 \bar{W}-W-i)
$$

Solving $W_{z}$ from (5.3) we obtain

$$
W_{z}=\frac{2[-i(W-\bar{W})]^{3 / 2}}{|A|^{2}-|\mu|^{2}|W-i|^{2}}\left[\bar{A} \Phi_{z}+i(W-i) \bar{\mu} \bar{\Phi}_{\bar{z}}\right]+R
$$

for some smooth function $\mathrm{R}$ of $W, \bar{W}, F$ and $\bar{F}$ in question which is also bounded since

$$
|s|=\frac{|W-i|}{|A|}=\frac{|W-i|}{|2 \bar{W}-W-i|} \leq q<1
$$

for some constant $q$ depending only on $1 / \min K$, and $\theta$ in (1.7) since $\operatorname{Im} W$ is strictly positive. Combining (5.2) with (5.4) provides

$$
\Phi_{\bar{z}}=Q_{1} \Phi_{z}+Q_{2} \bar{\Phi}_{\bar{z}}+R
$$

where

$$
Q_{1}=\frac{\mu\left[|A|^{2}-|W-i|^{2}\right]}{|A|^{2}-|\mu|^{2}|W-i|^{2}}, Q_{2}=i A(W-i) \frac{\left[|\mu|^{2}-1\right]}{|A|^{2}-|\mu|^{2}|W-i|^{2}}
$$

and $\mathrm{R}$ is another bounded smooth function of $W, \bar{W}, F$ and $\bar{F}$ in question. Now let us show

$$
\left|Q_{1}\right|_{\infty}+\left|Q_{2}\right|_{\infty} \leq q_{1}<1
$$

for some constant $q_{1}$ depending only on $1 / \min K$, and $\theta$ in (1.7). Indeed, by the definition in (5.5)

$$
\begin{aligned}
\left|Q_{1}\right|+\left|Q_{2}\right| & \leq \frac{|\mu|\left(1-|s|^{2}\right)+|s|\left(1-|\mu|^{2}\right)}{1-|s|^{2}|\mu|^{2}}=|\mu|+\frac{\left(1-|\mu|^{2}\right)|s|(1-|s||\mu|)}{1-|s|^{2}|\mu|^{2}} \\
& \leq|\mu|+(1-|\mu|) \frac{(1+|\mu|)|s|}{1+|s||\mu|}=1-\frac{1}{2}(1-|\mu|)(1-|s|)
\end{aligned}
$$

This implies that we can take $q_{1}=1-\frac{1}{2}\left(1-|\mu|_{\infty}\right)(1-q)<1$. 
LEMMA 5.1. Suppose that $W$ is a smooth solution to the problem (3.6)(3.9) and that there are two positive constants $d$ and $\delta$ such that

$$
\frac{H}{\sqrt{K}}-1 \geq d \text { for all } u^{2}+v^{2} \geq 1-\delta^{2}
$$

Then $\left\|D^{2} W\right\|_{p} \leq C_{p}$ for all $p \in(2,+\infty)$ and $|D W|_{\alpha},|D H|_{\alpha} \leq C_{\alpha}$ for each $\alpha \in(0,1)$ where $C_{p}$ and $C_{\alpha}$ depending only on $1 / \min K, 1 / d, 1 / \delta, \theta$ in (1.7), $|g|_{2},|K|_{2}$ and $|h|_{2}$.

Proof. Lemma 3.4 provides the bound for the mean curvature and hence, the bounds of $|W|_{\infty}, 1 /|W-\bar{W}|_{\infty}$. By means of the technique of cutoff functions we can easily obtain the interior estimates for solutions to (3.6) (3.9). namely, with $D_{\delta / 2}=\left\{u^{2}+v^{2}<1-\delta^{2} / 4\right\}$,

$$
\left\|D^{2} W\right\|_{L p_{\left(D_{\delta / 2}\right)}} \leq C_{p} \text { and }|D W|_{C \alpha_{\left(\bar{D}_{\delta / 2}\right)}} \leq C_{\alpha}
$$

for each $p \in[2,+\infty)$ and $\alpha \in(0,1)$ and some constants $C_{p}, C_{\alpha}$ under control. Now we proceed to deal with the estimates near the boundary. Set $\phi=\frac{H}{\sqrt{K}}-1=|\Phi|^{2}$. An differentiation yields

$$
\phi_{\bar{z}}=\Phi_{\bar{z}} \bar{\Phi}+\Phi \bar{\Phi}_{\bar{z}} \text { and } \phi_{z}=\left(\bar{\Phi}+\Phi \bar{Q}_{2}\right) \Phi_{z}+\Phi \bar{Q}_{1} \bar{\Phi}_{\bar{z}}+\Phi \bar{R}
$$

Solving $\Phi_{z}$ from the second part of (5.11) and its conjugate we have

$$
\Phi_{z}=\frac{\phi_{z}\left(\Phi+\bar{\Phi} Q_{2}\right)-\phi_{\bar{z}} \Phi \bar{Q}_{1}+|\Phi|^{2}\left(R \bar{Q}_{1}-\bar{R} Q_{2}\right)-\Phi^{2} \bar{R}}{\left|\Phi+\bar{\Phi} Q_{2}\right|^{2}-|\Phi|^{2}\left|Q_{1}\right|^{2}}
$$

Differentiation of the first equation of (5.11) using (5.6) gives

$$
\begin{aligned}
\phi_{z \bar{z}} & =\left|\Phi_{z}\right|^{2}-\left|\Phi_{\bar{z}}\right|^{2}+\partial_{z}\left(\Phi_{\bar{z}} \bar{\Phi}\right)+\partial_{\bar{z}}\left(\Phi \bar{\Phi}_{z}\right) \\
& =\partial_{z}\left[\left(Q_{1} \Phi_{z}+Q_{2} \bar{\Phi}_{\bar{z}}\right) \bar{\Phi}\right]+\partial_{\bar{z}}\left[\left(\bar{Q}_{1} \bar{\Phi}_{\bar{z}}+\bar{Q}_{2} \Phi_{z}\right) \Phi\right]+R_{1}\left(\Phi_{z}, \bar{\Phi}_{\bar{z}}\right)
\end{aligned}
$$

where $R_{1}$ is a polynomial of $\Phi_{z}$ and $\bar{\Phi}_{\bar{z}}$ up to second degree with bounded coefficients. In getting the last expression we have used (5.4). Inserting (5.12) into (5.13) we derive the equation for $\phi$.

$$
\begin{aligned}
L(\phi) & =\phi_{z \bar{z}}+2 R e \partial_{z}\left(\frac{\phi_{\bar{z}}\left(|\Phi|^{2}\left|Q_{1}\right|^{2}-|\Phi|^{2}\left|Q_{2}\right|^{2}-\bar{\Phi}^{2} Q_{2}\right)-\phi_{z}|\Phi|^{2} Q_{1}}{\left|\Phi+\bar{\Phi} Q_{2}\right|^{2}-|\Phi|^{2}\left|Q_{1}\right|^{2}}\right) \\
& =R_{2}\left(\phi_{z}, \phi_{\bar{z}}\right) \text { in } D / D_{\delta}
\end{aligned}
$$

where $R_{2}$ is also a polynomial of $\phi_{z}$ and $\phi_{\bar{z}}$ up to second degree with bounded coefficients since

$$
\left|\Phi+\bar{\Phi} Q_{2}\right|^{2}-|\Phi|^{2}\left|Q_{1}\right|^{2} \geq|\Phi|^{2}\left(1-\left|Q_{1}\right|-\left|Q_{2}\right|\right)^{2} \geq d^{2}\left(1-q_{1}\right)^{2} \text { in } D / D_{\delta}
$$

Notice that (5.14) is uniformly elliptic since

$$
\begin{aligned}
& \left(\xi^{2}+\eta^{2}\right)\left(\left|\Phi+\bar{\Phi} Q_{2}\right|^{2}+|\Phi|^{2}\left|Q_{1}\right|^{2}-2 \operatorname{Re}\left(\bar{\Phi}^{2} Q_{2}\right)-2|\Phi|^{2}\left|Q_{2}\right|^{2}\right) \\
& \quad-2 \operatorname{Re}\left[(\xi-i \eta)^{2}\left(|\Phi|^{2} Q_{1}\right)\right] \\
\geq & \left(\xi^{2}+\eta^{2}\right)|\Phi|^{2}\left(1+\left|Q_{1}\right|^{2}+\left|Q_{2}\right|^{2}-2\left|Q_{1}\right|-2\left|Q_{2}\right|^{2}\right) \\
\geq & d\left(1-q_{1}\right)^{2}\left(\xi^{2}+\eta^{2}\right) \text { for all } \xi, \eta \in R^{1}
\end{aligned}
$$

Using the De Giorge iteration soon gives the Holder estimates of $\phi$ in $C^{\alpha}\left(\left(\bar{D} / D_{\delta}\right)\right)$ for some positive $\alpha$ depending only on the quantities mentioned in the present lemma. 
Combining the $L^{p}$ theory for elliptic boundary value problem with (5.10) and the Nirenberg's inequality we can soon obtain

$$
\left\|D^{2} \phi\right\|_{L} p_{\left(D / D_{\delta}\right)} \leq C_{p}
$$

for each $p \in(2,+\infty)$ and some constant $C_{p}$ under control. Now in view of (5.4) (5.10) (5.12) (5.15) the present lemma is readily proved.

The proof for Theorem C. Let $\vec{r}_{0}$ be radius symmetric and of no umbilical points on $\partial D$. Then Lemma 2.2 tells us the umbilical point index of $\vec{r}_{0}$ equal to -2 . Denote its mean curvature and complex Riemann invariant by $H_{0}$ and $W_{0}$. Suppose that there is a $C^{3}$ solution to problem (1.1) with the prescribed mean curvature $h$ on $\partial D$. Denote its corresponding mean curvature and complex Riemann invariant by $H$ and $W$. Set $\Delta \Phi=\Phi\left(W_{0}\right)-\Phi(W)$. Then from (5.6) it follows that

$$
(\Delta \Phi)_{\bar{z}}=Q_{1}(\Delta \Phi)_{z}+Q_{2}(\Delta \bar{\Phi})_{\bar{z}}+R_{1}\left(W_{0}-W\right)+R_{2}\left(\bar{W}_{0}-\bar{W}\right) \text { in } D
$$

for some bounded functions $R_{1}$ and $R_{2}$.

Now we are going to express $\left(W_{0}-W\right)$ in terms of $\Delta \Phi$. Notice that with $\Phi=$ $(W-i) / \sqrt{-i(W-\bar{W})}$

$$
\Phi-\Phi_{0}=\left[\Phi_{w}\right]\left(W-W_{0}\right)+\left[\Phi_{\bar{w}}\right]\left(\bar{W}-\bar{W}_{0}\right)
$$

where $\left[\Phi_{w}\right]=\int_{0}^{1} \Phi_{w}\left(\lambda W+(1-\lambda) W_{0}, \lambda \bar{W}+(1-\lambda) \bar{W}_{0}\right) d \lambda$ and so on. It is easy to see

$$
\operatorname{Re}\left[\Phi_{w}\right]=3 b_{1}+b_{2} \text { and } \operatorname{Re}\left[\Phi_{\bar{w}}\right]=b_{1}-b_{2}
$$

where

$$
\begin{aligned}
& b_{1}=\frac{1}{2^{5 / 2}} \int_{0}^{1} \frac{d \lambda}{\left[\lambda \operatorname{Im} W+(1-\lambda) \operatorname{Im} W_{0}\right]^{1 / 2}} \\
& b_{2}=\frac{1}{2^{5 / 2}} \int_{0}^{1} \frac{d \lambda}{\left[\lambda \operatorname{Im} W+(1-\lambda) \operatorname{Im} W_{0}\right]^{3 / 2}}
\end{aligned}
$$

Moreover, both of $b_{1}$ and $b_{2}$ have positively bounds from both sides. Hence

$$
\left|\left[\Phi_{w}\right]\right| \geq \frac{1}{C} \text { and } \frac{\left|\left[\Phi_{\bar{w}}\right]\right|}{\left|\left[\Phi_{w}\right]\right|} \leq 1-\frac{1}{C} \text { since } \operatorname{Im}\left[\Phi_{w}\right]=-\operatorname{Im}\left[\Phi_{\bar{w}}\right]
$$

for some constant $C$ under control. Solving $W-W_{0}$ from (5.17) we have

$$
W-W_{0}=\frac{\left(\Phi-\Phi_{0}\right)\left[\bar{\Phi}_{\bar{w}}\right]-\left(\bar{\Phi}-\bar{\Phi}_{0}\right)\left[\Phi_{\bar{w}}\right]}{\left|\left[\Phi_{w}\right]\right|^{2}-\left|\left[\Phi_{\bar{w}}\right]\right|^{2}}
$$

Obviously the coefficients of $\left(\Phi-\Phi_{0}\right)$ and $\left(\bar{\Phi}-\bar{\Phi}_{0}\right)$ are bounded by (5.18). Inserting (5.19) into (5.16) one can get

$$
(\Delta \Phi)_{\bar{z}}=Q_{1}(\Delta \Phi)_{z}+Q_{2}(\Delta \bar{\Phi})_{\bar{z}}+A_{1}(\Delta \Phi)+A_{2}(\Delta \bar{\Phi}) \text { in } D
$$

where $A_{1}, A_{2}$ are bounded from both sides. The theory on the generalized analytic functions tells us

$$
(\Delta \Phi)=\chi(\zeta) \exp R_{1}
$$

where $\zeta$ is a Holder continuous homeomorphism from the unit disk onto itself, constructed in Lemma 4.1 and

$$
R_{1}=T\left[A_{1}+A_{2} \frac{\left(\bar{\Phi}-\bar{\Phi}_{0}\right)}{\left(\Phi-\Phi_{0}\right)}\right] \text { where } \operatorname{Re}\left(R_{1}\right)=0 \text { on } \partial D
$$


From

$$
|\Delta \Phi| \geq\left|\Phi\left(W_{0}\right)\right|-|\Phi(W)| \geq \sqrt{\frac{H_{0}}{\sqrt{K}}-1}-\sqrt{\frac{h}{\sqrt{K}}-1}>0 \text { on } \partial D
$$

it follows that the winding number Index $(\chi)=\operatorname{Index}(\Delta \Phi)=\operatorname{Index}\left(\Phi\left(W_{0}\right)\right)=$ Index $\left(W_{0}-i\right)=\operatorname{Index}\left(\vec{r}_{0}\right)=-2$. This contradicts to the fact that $\chi$ is analytic in the unit disk and Theorem $\mathrm{C}$ is proved.

The proof for Theorem A. Suppose that there is a smooth isometric immersion $\vec{r}_{0}$ of the given positive disk. Denote its mean curvature by $H_{0}$. By the hypothesis on the given prescribed mean curvature (1.6) one can find an extension of $h$ in $C^{\infty}(\bar{D})$, still denoted by $h$, subject to (4.17)(4.18). Let the given metric $g_{\lambda}$ be defined in (4.16). Consider the problem (1.1) for $\left(\bar{D}, g_{\lambda}\right)$ with the prescribed mean curvature $h(\lambda u)$ on $\partial D$ and the subsidiary condition (3.32)(4.19). Theorem 3.3 and Lemma 4.3 tell us that the following set

$$
\Lambda=\left\{\lambda \in\left[\lambda_{0}, 1\right] \mid(1.1) \text { has a solution in } C^{\infty}(\bar{D}) \text { with }(3.23)(4.19)\right\}
$$

is open as well as not empty for some $\lambda_{0}>0$.

In what follows we shall prove $\Lambda$ close. Evidently, $\vec{r}_{0}(\lambda u)$ is a smooth isometric immersion of $\left(\bar{D} \cdot g_{\lambda}\right)$ as $\lambda>0$. Denote the complex Riemann invariants of $\vec{r}_{0}$ by $W_{0}$. It is easy to see $W_{0}(\lambda u)$ being the complex Riemann invariants of $\vec{r}_{0}(\lambda u)$. As $\lambda \in \Lambda$, denote the corresponding solution and complex Riemann invariants by $\vec{r}$ and $W$. Analogously, set $\Phi(W)=\Phi, \Phi\left(W_{0}\right)=\Phi_{0}$. Lemma 3.4 tells us $|W|_{\infty}, 1 / \operatorname{Im} W$ and $|\Phi|_{\infty}$ bounded above by a constant $C$ independent of $\lambda$. Now we are in a position to show that for some two positive constants $d$ and $\delta$ independent of $\lambda$,

$$
\frac{H}{\sqrt{K}}-1=|\Phi|^{2} \geq d^{2} \text { as } u^{2}+v^{2} \geq 1-\delta^{2}
$$

With $\Phi-\Phi_{0}=\Delta \Phi$ using (5.6) we have

$$
\partial_{\bar{z}}(\Delta \Phi)=Q_{1} \partial_{z}(\Delta \Phi)+Q_{2} \partial_{\bar{z}}(\Delta \Phi)+A_{1}(\Delta \Phi) \text { in } D
$$

for some function $A_{1}$ uniformly bounded. A similar argument gives (5.20) for $\Delta \Phi$. From

$$
\begin{aligned}
|W-i| & =\left|W-\frac{h}{\sqrt{K}} i-\left(1-\frac{h}{\sqrt{K}}\right) i\right| \geq \sqrt{\left(\frac{h}{\sqrt{K}}\right)^{2}-1}-\left(\frac{h}{\sqrt{K}}-1\right) \\
& \geq \sqrt{\left(\frac{h}{\sqrt{K}}\right)-1}\left[\sqrt{\left(\frac{h}{\sqrt{K}}\right)+1}-\sqrt{\left(\frac{h}{\sqrt{K}}\right)-1}\right]>0 \text { on } \partial D
\end{aligned}
$$

it follows that

$$
\operatorname{Index}(\Phi)=\operatorname{Index}(W-i)=\operatorname{Index}\left(W-\frac{h}{\sqrt{K}} i\right)=n
$$

Distinguish two cases.

Case a. $n=0$, Since $\left|\Phi-\Phi_{0}\right| \geq|\Phi|-\left|\Phi_{0}\right|>0$ on $\partial D$ by (4.17), it turns out Index $(\chi)=$ Index $(\Phi)=0$, By the analyticity of $\chi$ we know $\chi$ of no null points in $D$. Hence, an application of the maximum principle to $\chi$ noting $|\Phi|^{2}=\frac{H}{\sqrt{K}}-1$ yields

$$
|\chi| \geq \min _{\partial D}\left|\Phi-\Phi_{0}\right| \geq\left(1+\epsilon_{0}\right)\left(\max _{\partial D}\left|\Phi_{0}\right|+\epsilon_{0}\right) \text { in } D
$$


for some positive constant $\epsilon_{0}$. Consequently, for some $\alpha \in(0,1)$,

$$
\begin{aligned}
|\Phi(q)| \geq|\chi|\left|\exp R_{1}\right|-\left|\Phi_{0}\right| \geq\left[1-C_{1}(1-|q|)^{\alpha}\right]\left(1+\epsilon_{0}\right)\left(\max _{\partial D}\left|\Phi_{0}\right|+\epsilon_{0}\right) \\
-\left[1+C_{2}(1-|q|)\right] \max _{\partial D}\left|\Phi_{0}\right| \geq \frac{1}{2} \epsilon_{0}\left(\max _{\partial D}\left|\Phi_{0}\right|+\epsilon_{0}\right) \text { as }|q| \geq 1-\delta
\end{aligned}
$$

for some small positive $\delta$ independent of $\lambda$. In getting (5.25) we have used the following property of $R_{1}$ from (5.21), i.e., $\left\|D R_{1}\right\|_{p} \leq C_{p}, p>2$, close to 2 and for some constant $C_{p}$ independent of $\lambda$. By the definition of $\Phi(5.23)$ is readily proved for the case $n=0$.

Case b. $n>0$. Under the present circumstance Index $(\chi)=n>0$ and $\chi$ is of $n$ null points in $D$. Notice that $\Phi\left(p_{k}\right)=\Phi_{0}\left(p_{k}\right), k=1,2, . ., n$. Set

$$
\chi_{0}=e^{i \alpha} \frac{\zeta-\zeta\left(p_{1}(t)\right)}{1-\bar{\zeta}\left(p_{1}(t)\right) \zeta} \frac{\zeta-\zeta\left(p_{2}(t)\right)}{1-\bar{\zeta}\left(p_{2}(t)\right) \zeta} \ldots \frac{\zeta-\zeta\left(p_{n}(t)\right)}{1-\bar{\zeta}\left(p_{n}(t)\right) \zeta}
$$

where $\zeta$ is constructed in Lemma 4.1. Then $\chi_{0}$ is analytic in $\zeta$. Since Index $(\chi)=n$, by the Blaschke decomposition we have $\chi=\chi_{0} \chi_{1}$ where $\chi_{1}$ is also analytic and no null points in $D$. By the maximum principle it turns out

$$
\left|\chi_{1}(p)\right| \geq \min _{\partial D}\left(|\Phi|-\left|\Phi_{0}\right|\right) \geq\left(1+\epsilon_{0}\right)\left(\max _{\partial D}\left|\Phi_{0}\right|+\epsilon_{0}\right) \text { for all } p \in D
$$

In view of the explicit formula of $\chi_{0}$ we know $\left|\chi_{0}(p)\right| \geq[1-C \delta]$ as $|p| \geq 1-\delta$ for another positive constant $\delta$ independent of $\lambda$. Now we can conclude that

$$
\begin{aligned}
|\Phi(p)| & \geq\left|\exp R_{1}\left(\chi_{0} \chi_{1}\right)\right|-\left|\Phi_{0}\right| \\
& \geq(1-C \delta)\left(1-C \delta^{\alpha}\right)\left(1+\epsilon_{0}\right)\left(\max _{\partial D}\left|\Phi_{0}\right|+\epsilon_{0}\right)-(1+C \delta) \max _{\partial D}\left|\Phi_{0}\right| \\
& \geq \frac{1}{2} \epsilon_{0}\left(\max _{\partial D}\left|\Phi_{0}\right|+\epsilon_{0}\right) \text { as }|p| \geq 1-\delta
\end{aligned}
$$

if $\delta$ is sufficiently small. So (5.23) follows immediately.

The remaindering part of the proof for the closeness can be directly completed by means of Lemma 5.1 without difficulties.

The proof for the uniqueness. Suppose that (1.1) with (1.5) admits at least three solutions. Then without loss of generality we may assume that there are at least two solutions $\vec{r}_{i} \in C^{\infty}\left(\bar{D}, R^{3}\right) i=1,2$ such that their corresponding complex Riemann invariants satisfy

$$
W_{i}=\frac{h}{\sqrt{K}}+i \sqrt{\frac{h^{2}}{K}-1} \text { at } p_{0}=(1,0)
$$

Extend the prescribed mean curvature $h$ subject to (4.17)(4.18). Scaling the metric $g$ by $g_{\lambda}$ like (4.16). Consider the set (5.22) for $\lambda_{0}=0$ and evidently, $\lambda=1 \in \Lambda$. Let us study the continuation from $\lambda=1$ to $\lambda=0$. In a similar argument in proving the existence of Lemma 4.3 and by means of the previous arguments of proving openness and closeness of set $S$ we can find two families of solutions $W_{i}(\lambda) \in C^{1}\left([0,1], C^{\infty}\left(\bar{D}, R^{3}\right)\right)$ $i=1,2$. The uniqueness of solutions to the problem (4.6)-(4.8) tells us $W_{1}(0)=W_{2}(0)$. And with the aid of the contraction mapping principle it follows that $W_{1}(\lambda)=W_{2}(\lambda)$ and hence, $\vec{r}_{1}(\lambda)=\vec{r}_{2}(\lambda)$ as $\lambda \in\left(0 . \lambda_{*}\right]$ for some positive $\lambda_{*}$. Then repeated finite same arguments we can easily deduce $\vec{r}_{1}(1)=\vec{r}_{2}(1)$. This completes the proof by contradiction.

Now we proceed to deal with the proof for the last part of Theorem A. Let us focus our attention on the spherical map from $\vec{r}$ into $S^{2}$. If it is diffeomorphic, then $\vec{r}$ 
is a convex surface and an embedding from $\bar{D}$ into $R^{3}$. Since $\vec{r}$ has positive curvature, it follows that its spherical map is an immersion into $S^{2}$. Suppose that there are two points $q_{1}, q_{2} \in \bar{D}$ whose the images of the spherical map of $\vec{r}\left(q_{1}\right)$ and $\vec{r}\left(q_{2}\right)$ coincide with each other and without less of generality, we may assume that the image is just the unit vector of the $z$ axis, $\vec{k}$. By the hypothesis that the geodesic curvature of $\partial D$ is nonnegative, one can find an arc length parameterized geodesic $\gamma(s)$ connecting $\gamma(0)=\vec{r}\left(q_{1}\right)$ and $\gamma(l)=\vec{r}\left(q_{2}\right)$. Denote the image of the spherical map along $\gamma(s)$ by $\vec{n}(s)$. Then for each $s \in[0, l]$, without loss of generality, we may assume $s \leq l / 2$ (otherwise $l-s \leq l / 2$ ),

$$
\begin{aligned}
d i s_{s^{2}}(\vec{n}(s) \cdot \vec{n}(0)) & \leq \int_{0}^{s} \sqrt{2 H I I\left(\frac{d u}{d s}, \frac{d v}{d s}\right)-K g\left(\left(\frac{d u}{d s}, \frac{d v}{d s}\right)\right.} d s \\
& \leq \int_{0}^{s}\left\{H+\sqrt{H^{2}-K}\right\} d s \\
& \leq \frac{d}{2} \max \left\{\theta+\sqrt{\theta^{2}-K}\right\} \leq \frac{\pi}{2}
\end{aligned}
$$

This implies that the images of the spherical map of $\gamma(s), s \in[0, l]$ is contained in the below hemisphere. Noting that the Gauss curvature of $\vec{r}$ is positive everywhere we can see the vertical component of $\vec{r}, z(\gamma(s))=z(s)$ satisfying

$$
z_{s s}>0 s \in(0, l)
$$

On the other hand $\vec{k}$ is the normal to $\vec{r}$ at $\gamma(0)$ and $\gamma(l)$ and hence, $z_{s}(0)=z_{s}(l)=0$. this contradicts to (5.29) and proves the previous claim. This ends the proof for the main theorem.

\section{REFERENCES}

[A] L. Ahlfors, Lectures on Quasiconformal Mappings, Princeton, 1966.

[DE] Ph. Delanoe, Relations globalement regulieres de disques strictement convexes dans les espaces d'Euclide et de Minkowski par la methode de Weingarten, Ann. Sci. Ec Norm. Super., IV, 21 (1988), pp. 637-652.

[G] M. Gromov, Partial Differential Relations, Springer-Verlage, Berlin Heidelberg, 1986.

[GR] M. Gromov And V. A. Rohklin, Embeddings and immersions in Riemannian geometry, Russian Mathematical Surveys, 25 (1970), pp. 1-57.

[GI] M. Giaquinta, Multiple Integrals in the Calculus of Variations and Nonlinear Elliptic Systems, Princeton University Press, Princeton, 1983.

[GY] F. GUAN AND Y. LI, The weyl problem with nonnegative Gauss curvature, J. Diff. Geometry, 39 (1994), pp. 331-342.

[HO1] J. X. HONG, Isometric embedding in $R^{3}$ of complete noncompact nonnegatively curved surfaces, Manuscripta Math., 94 (1997), pp. 271-286.

[HO2] J. X. Hong, Dirichlet problems for general Monge-Ampere equations, Math. Z., 209 (1992), pp. 289-306.

[HO3] J. X. Hong, Darboux equations and isometric embedding of Riemannian manifolds with non negative curvature in $R^{3}$, Chin. Ann. of Math., 20 (1999), pp. 123-136.

[HZ] J. X. HONG AND C. ZUILY, Isometric embedding of the 2-sphere with non negative curvature in $R^{3}$, Math. Z., 219 (1995), pp. 323-334.

[IA] J. A. IAIA, Isometric embeddings of surfaces with nonnegative curvature in $R^{3}$, Duke Math. J., 67 (1992), pp. 423-459.

[LE1] H. LEWY, On the existence of a closed convex surface realizing a Riemannian metric, Proc. Nat. Acad. Sci., 24 (1936), pp. 104-106.

[NI1] L. Nirenberg, The Weyl and Minkowski problems in differential geometry in the large, Comm. Pure Appl. Math., 6 (1953), pp. 337-394. 
[NI2] L. Nirenberg, in Nonlinear Problems, R. E. Langer, ed., University of Wisconsin Press, Madison, 1963, pp. 177-193.

[PO] A. V. Pogorelov, Extrinsic Geometry of Convex Surfaces, Transl. Math. Monogr. 35, Providence, RI, Am.Math.Soc., 1973.

[V] I. N. Vekua, Generalized Analytic Functions, Fizmatgiz, Moscow, 1959; English transl Pergamon Press, London, 1962.

[W] H. Whitney, Differentiable Manifolds, Ann. of Math, 37:2 (1936), pp. 645-680.

[Y1] S. T. YAU, Problem Section, Seminar on Differential Geometry, Princeton University Press, 1982.

[Y2] S. T. YAu, Open Problems in Geometry, Chern-A Great Geometer on the Twentith Century, International Press, 1992.

[Y3] S. T. YAU, Lecture on Differential Geometry, in Berkeley, 1977.

[Y4] S. T. YAU, Survey on Geometry and Analysis, Asian J. Math., 4 (2000), pp. 235-278. 